\title{
Dynamic Distortions in the HARP TPC: observations, measurements, modelling and corrections
}

\author{
A. Bagulya ${ }^{\text {a }}$ A. Blondel ${ }^{\text {b }}$ S. Borghi ${ }^{\text {b,c }}$ G. Catanesi ${ }^{\mathrm{d}}$ \\ P. Chimenti ${ }^{\text {e }}$ U. Gastaldi ${ }^{\mathrm{f}}$ S. Giani ${ }^{\mathrm{g}, *}$ V. Grichine ${ }^{\mathrm{h}}$ \\ V. Ivanchenko ${ }^{g, i}$ D. Kolev ${ }^{j}$ J. Panman $^{\mathrm{g}}$ E. Radicioni ${ }^{\mathrm{d}}$ \\ R. Tsenov $^{j}$ I. Tsukerman ${ }^{k}$ \\ ${ }^{a}$ Institute for Nuclear Research, Moscow, Russia \\ b Université de Genève, Switzerland \\ c Current address University of Glasgow, UK \\ ${ }^{\mathrm{d}}$ INFN, Bari, Italy \\ e INFN, Trieste, Italy \\ ${ }^{\mathrm{f}}$ Laboratori Nazionali di Legnaro dell INFN, Legnaro, Italy \\ g CERN, Geneva, Switzerland \\ ${ }^{\mathrm{h}}$ P. N. Lebedev Institute of Physics (FIAN), Russian Academy of Sciences, \\ Moscow, Russia \\ ${ }^{\mathrm{i}}$ On leave of absence from Ecoanalitica, Moscow State University, Moscow, Russia \\ ${ }^{\mathrm{j}}$ Faculty of Physics, St. Kliment Ohridski University, Sofia, Bulgaria \\ ${ }^{\mathrm{k}}$ ITEP, Moscow, Russian Federation
}

\begin{abstract}
The HARP experiment was designed to study hadron production in protonnucleus collisions in the energy range of $1.5 \mathrm{GeV} / c-15 \mathrm{GeV} / c$. The experiment was made of two spectrometers, a forward dipole spectrometer and a large-angle solenoid spectrometer. In the large-angle spectrometer the main tracking and particle identification is performed by a cylindrical Time Projection Chamber (TPC) which suffered a number of shortcomings later addressed in the analysis. In this paper we discuss the effects of time-dependent (dynamic) distortions of the position measurements in the TPC which are due to a build-up of ion charges in the chamber during the accelerator spill. These phenomena have been studied both theoretically and experimentally, and a correction procedure has been developed. First, the dynamics of the positive ion cloud and of the full electrostatics of the field-cage system have been modelled with a phenomenological approach and a general correction procedure has been developed and applied to all data settings. Then, the correc-
\end{abstract}


tion procedure has been benchmarked experimentally by means of recoil protons in elastic scattering reactions, where the track coordinates are precisely predictable from simple kinematical considerations. After application of the corrections for dynamic distortions the corrected data have a performance equal to data where the dynamic distortions are absent. We describe the theoretical model, the comparison with the measurements, the distortion correction method and the results obtained with experimental data.

\section{Introduction}

The HARP experiment (1; 2) was designed to study hadron production in proton-nucleus collisions in the energy range of $1.5 \mathrm{GeV} / c-15 \mathrm{GeV} / c$. The main aim of the experiment is to provide pion production data for the calculation of neutrino fluxes in conventional neutrino beams at accelerators, to provide data for extended air shower simulations and for prediction of the atmospheric neutrino flux, as well as to provide input to the quantitative design of a future neutrino factory.

The experiment was made of two spectrometers:

- A forward dipole spectrometer with planar drift chambers for the particle tracking and a time-of-flight (TOF) scintillator wall, a Cherenkov detector and an electromagnetic calorimeter for particle identification (PID).

- A large-angle solenoid spectrometer where the main tracking and PID is performed by a cylindrical Time Projection Chamber (TPC) occupying most of the radial space of the solenoid magnet. The TPC provides track, momentum and vertex measurements for all outgoing charged particles in the angular range from $20^{\circ}$ to $135^{\circ}$ with respect to the beam axis. In addition, it provides particle identification by recording the particle's energy loss in the gas $(\mathrm{d} E / \mathrm{d} x)$. The PID capabilities of the TPC detector are complemented by a set of multi-gap RPCs (Resistive Plate Chambers) serving as TOF detectors and surrounding the TPC.

Data analysed with the large-angle spectrometer have been published in Refs. $(3 ;$; $;$; 5 ; 6 )

\section{The HARP TPC}

The schematic layout of the HARP TPC is shown in Fig. 1. The TPC is positioned inside the solenoid magnet, providing a magnetic volume with a diameter of $0.9 \mathrm{~m}$, a length of $2.25 \mathrm{~m}$ and a field of $0.7 \mathrm{~T}$ in the main sensitive volume. The magnet was previously used for the R\&D of the ALEPH experiment's TPC (7) and later modified for HARP. The downstream end of the return yoke was left open to minimize materials encountered by secondary particles emerging from the TPC in the direction of the forward spectrometer. At the upstream end there is a small cylindrical hole in the end-cap yoke for the passage of

\footnotetext{
* Corresponding author e-mail: Simone.Giani@cern.ch
} 
the incident beam and to leave space to insert the inner trigger cylinder (ITC) and target holder inside the inner field cage (IFC). The drift volume is $1541 \mathrm{~mm}$ long with a nominal electric field gradient of $111 \mathrm{~V} / \mathrm{cm}$. Given the drift velocity of the chosen gas mixture under these operating conditions, the maximum drift time is approximately $30 \mu \mathrm{s}$. The induced charge from the gas amplification at the anode wires is measured using a pad plane, subdivided in six sectors; the anode wires are strung onto the six spokes defining the sectors. The pads are organized in 20 concentric rows, each pad being connected to an individual pre-amplifier. The pad dimensions are $6.5 \mathrm{~mm} \times 15 \mathrm{~mm}$ and the number of pad ranges from 11 per row per sector at the inner radius to 55 at the outer radius. The pad-charges are sampled into charge time-series by one Flash-ADC (FADC) per pad, with a sampling interval of 100 ns. The total Data Acquisition (DAQ) readout time is $500 \mu$ s to $1000 \mu$ s per event depending on the event size.

During the analysis, after unpacking the FADC values, time-series are organized in $R \phi$ clusters. The clusters are assigned to tracks by a tree-based algorithm for pattern recognition which used a general three-dimensional binary search method for fast look-up of clusters (8).

Once clusters are assigned to a track, a helix fit is performed. The fitting procedure is based on the algorithm developed by the ALEPH Collaboration (9) with slight modifications $(10)$, e.g. the possibility to fit tracks which spiral for more than $2 \pi(11)$. The fit consists of two consecutive steps: a circle-fit in the $x-y$ plane 1 , based on a least-square method (12), and a subsequent straight line fit in the $z-s_{x y}$ plane 2 . The two fitting steps allow the five parameters which uniquely define the helix to be determined. The code uses the same naming and sign conventions as in the TASSO and ALEPH software (9) with a particle direction associated to the motion along the helix itself.

The analysis revealed that the TPC suffered from a number of operational problems which were discovered, one after the other, during and after the data taking:

(1) large excursions of the gains of the pad pre-amplifiers;

(2) a relatively large number of dead or noisy pads;

(3) large pad gain variations with time;

(4) static distortions caused by the inhomogeneity of the magnetic field, an accidental HV mismatch between the inner and outer field cage;

(5) cross-talk between pads caused by capacitive coupling between signal lines in the multilayer printed boards;

(6) dynamic distortions caused by build-up of ion-charge density in the drift volume during the $400 \mathrm{~ms}$ long beam spill.

The corrections for the first four effects are described in Refs. (2; 3; 11). A detailed discussion of the cross-talk effect can be found in Ref. (13). The dynamic distortion effect and its corrections are presented in this paper.

1 The Cartesian coordinates $x$ and $y$ are the coordinates perpendicular to the nominal magnetic field.

2 The $s_{x y}$ coordinate is defined as the arc length along the circle in the $x-y$ plane between a point and the impact point. 


\section{Evidence for TPC Dynamic Distortions and overall characteristics}

In a situation where an incoming beam hits a target at the centre of a rotationally symmetric TPC one expects the tracks of produced particles to a good approximation to emerge from the centre of the TPC. We then define $d_{0}$ to be the impact point of the tracks in the $x y$ plane, i.e. the minimum distance between the track and the $z$-beam axis in the $x y$ plane. By convention, its sign indicates whether the helix encircles the $z$-beam axis (positive sign) or not (negative sign). The distribution of $d_{0}$ is then expected to be symmetric around the origin. The presence of distortions in the TPC can modify this distribution. Thus, the distribution of the distance of closest approach of the helix to the nominal axis of the TPC is a measure of distortions in the TPC. The $d_{0}$ distribution of TPC tracks was found to show a difference between the peaks for tracks with opposite curvature, wih a separation depending on the beam tuning and intensity (11). This effect is shown in Fig. 2. The two panels of the figure show two different runs of the same setting ( $8 \mathrm{GeV} / c$ on a $5 \% \lambda_{\mathrm{I}}$ Be target) taken close to each other in time, one just before and the other after re-tuning of the beam.

The presence of the effect persisted even after correcting for static distortion due to voltage misalignment between the inner and outer field cages, the effect of which was shown to be much less important.

A better measure of the impact parameter, namely the track impact distance with respect to the trajectory of the incoming beam particle, $d_{0}^{\prime} 3$, was found to be a very sensitive probe to measure the distortion strength. The difference between $d_{0}^{\prime}$ and $d_{0}$ may be large due to the relatively large width of the beam spot at the target $(\approx 5 \mathrm{~mm})$ and due to the fact that the beam was not always centred.

The influence of the distortions can be monitored using its average value $\left\langle d_{0}^{\prime}\right\rangle$ as shown in Ref. (11). In Fig. 3 this quantity is displayed separately for positively and negatively charged pion tracks as a function of the event number within the spill $N_{\text {evt }}$. A similar benchmark was used in Ref. (14) for distortions observed in the STAR TPC. Due to the sign-convention, the dynamic distortions shift the $d_{0}^{\prime}$ value for particle tracks of positive and negative charge in opposite direction. The dependence of $d_{0}^{\prime}$ shows that a distortion effect builds up during the spill, testifying its dynamic character. The absence of distortions at the beginning of the spill, and the increasingly larger distortions with opposite sign for oppositely charged particles during the spill, explains the peak structure initially observed in the $d_{0}$ distribution. The curvature of high momentum tracks changes sign and therefore their $d_{0}^{\prime}$ migrates to large values with opposite sign.

The evolution of the $d_{0}^{\prime}$ distortion during the spill suggests a slowly drifting cloud of positive ions to be the cause of the track distortions. In addition, the absence of a saturation plateau suggests that the ion build-up does not reach its maximum before the end of the spill. It is therefore expected that a cloud of positive ions generated around the wires of the anode grid of the TPC grows progressively with time during the spill. The ion cloud would have approximately a torical shape with inner and outer diameter limited by the

$\overline{3}$ The $d_{0}^{\prime}$ sign indicates if the helix encircles the beam particle trajectory (positive sign) or not (negative sign) 
inner and outer field cages of the TPC. The front of the ion cloud does not reach the TPC $\mathrm{HV}$ cathode before the end of the spill. At the end of the beam spill the production of positive ions stops. During the inter-spill time the full cloud drifts to the HV cathode and vanishes completely before the start of the following spill. The minimum time between spills is ensured to be $2 \mathrm{~s}$ by the operation of the PS.

It was observed that the overall effect of the dynamic distortion in $R \phi$ was opposite in sign to the one induced by the static distortions due to the aforementioned HV mismatch. Via a straightforward $E \times B$ calculation it is possible to conclude that the radial component of the electric field due to dynamic distortions has to be directed predominantly outwards.

The hypothesis that dynamic distortions are caused by the build-up of positive ions in the drift volume during the $400 \mathrm{~ms}$ long beam spill makes it easier to understand why changes in the beam parameters (intensity, steering, focus) cause an increase or decrease in the dynamic distortions: the large amount of material around the target is likely to produce many low-energy secondary particles very close to the inner field cage whenever it is hit by a sizable beam halo. After the end of the beam spill, the initial conditions are re-established by the fact that the inter-spill time is large enough to drain all ions to the HV cathode.

Although charge build-up is a common phenomenon in TPCs, in the case of HARP the large amount of material before and around the target - very close to the beam axis - and the way the beam has often been tuned (too intense and/or not enough collimated) in some of the setting makes its occurrence rather difficult to deal with. In addition, the strongly inhomogeneous distortion of the electric field (larger at smaller radius) complicates the dynamic modelling of the actual field lines and of the ionization charge trajectories.

\section{Benchmarking the distortion effects using elastic scattering}

Elastic scattering interactions of protons and pions on hydrogen provide events where the kinematics are fully determined by the scattering angle of the forward scattered beam particle. These kinematic properties were exploited to provide a known beam of protons pointing into the TPC sensitive volume. Data sets taken with liquid hydrogen targets at beam momenta from $3 \mathrm{GeV} / c$ to $8 \mathrm{GeV} / c$ were used for this analysis.

A good fraction of forward elastically-scattered protons or pions enter into the acceptance of the forward spectrometer, where the full kinematics of the event can be constrained. In particular, the direction and momentum of the recoil proton can be precisely predicted. Selecting events with one and only one track in the forward direction and requiring that the measured momentum and angle are consistent with an elastic reaction already provides an enriched sample of elastic events. By requiring that only one barrel RPC hit is recorded at the position predicted for an elastic event (the precision of the prediction from the forward spectrometer is within the RPC pad size) and within a time window consistent with a proton time-of-flight, we obtained a $\simeq 99 \%$ pure sample of recoil protons in the TPC volume and with known momentum vector.

At beam momenta in the range $3 \mathrm{GeV} / c-8 \mathrm{GeV} / c$ the protons which are tagged by ac- 
cepted forward beam particles point into the TPC with angles of $\approx 70^{\circ}$ with respect to the beam direction. The beam counters provide a direct measurement of the incoming particle direction and of the scattering vertex coordinates in the target transverse plane. Once a clean sample of elastic-scattering events is isolated, by using detectors that are all independent from the TPC, the absolute efficiency of the track finding and fitting procedure can be measured (this allows the Monte Carlo calculations of the TPC detection efficiency to be benchmarked), and both the direction of emission and the momentum at the scattering vertex of the proton which traverses the TPC at large angle are determined for each event. After correction for energy loss and multiple scattering the complete track trajectory is determined. It can be used both for comparison with the track points of the proton track reconstructed from the TPC data not corrected for distortions, to measure directly the effect of dynamic distortions and for benchmarking the proton track reconstructed from TPC data after corrections of dynamic distortions (15). This fact has been used for a direct measurement of the distortions, as it will be described in Sec. 6 .

By comparison with the momentum vector predicted with the elastic scattering kinematics, it was verified with the data that the value of the polar angle $\theta$ is not modified by the dynamic distortions. However, the momentum measurement is affected. By disregarding the impact point of the incoming beam particle during the fit, the curvature of the track in the TPC gas volume can be measured directly. One observes that the momentum and the value of $d_{0}^{\prime}$ are biased as a function of $N_{\text {evt }}$ as shown in Fig. 4 . The $N_{\text {evt }}$ dependence for $d_{0}^{\prime}$ does not show a significant difference between the lower and higher momentum part of the spectrum.

The analysis of the elastic scattering events sets very stringent constraints on the maximum effect of distortions of all kinds on the measurements of kinematic quantities with the TPC. This method has provided solid estimates of the systematic errors associated with distortions as a function of event-in-spill. In particular, it has been used to estimate the overall systematic error on the momentum determination (3), (16).

\section{Ion Cloud Distortion Dynamics}

Given the beam intensity and the data acquisition rate with the $5 \%$ interaction length targets, it follows that HARP operated under conditions of high dead time (higher than $90 \%$ ). Hence, within one setting, the parameter $N_{\text {evt }}$ is a good measure of the time the event was taken after the start of the spill. (Depending on the precise data-taking conditions, the DAQ recorded about one event per ms.)

The beam instrumentation allows a precise evaluation of the direction, intensity and particle type of the impinging particles to be made. It is therefore possible to show correlations of beam properties and dynamic distortions. The beam is poorly focused during several setting 4 . It is observed that a badly tuned beam, with large number of halo particles which hit the target support material (representing several nuclear interaction lengths $\lambda_{\mathrm{I}}$ ) induces a large number of particles during the data-acquisition dead time. The contrary

4 HARP uses the word setting to define a group of runs with the same beam momentum and polarity, target and trigger definition. 
also holds: dynamic distortions disappear or are strongly reduced for settings where the beam is well focused, and can change from run to run during a setting when the beam was re-tuned between these runs. Moreover, it is observed that the dependencies of the distortions on the azimuthal angle $\phi$ (observed for some settings) are correlated with an off-axis tuning of the beam.

Beam particles hitting the beam entrance hole and the target support material produce secondaries which enter the TPC and produce ionization charges. Notice that a large number of low-energy (and therefore highly ionizing) electrons are expected to enter the active gas volume. This kind of phenomenon is likely to produce many more electron-ion pairs than the typical triggered event in the target. The produced ionization electrons drift towards the amplification region and then their number is multiplied near the pad plane with an amplification factor of the order of $10^{5}$, producing an equivalent number of argon ions. Any inefficiency of the gating grid at the level of $10^{-3}$ or even $10^{-4}$ allows an overwhelming number of ions to reach the drift region and to start travelling in the TPC gas volume towards the cathode, forming at the same time a positive charge cloud. This charge cloud distorts the (otherwise uniform) drift field.

From measurements of positive ion mobility in argon based gas mixtures (17), the velocity of the ions is computed to be about $2 \mathrm{~mm} / \mathrm{ms}$ (four orders of magnitude lower than the velocity of drift electrons in the TPC).

To help visualize the build-up and motion of the ion cloud, one should keep in mind that the pad plane is at $z \approx-500 \mathrm{~mm}$, the thin targets are at $z \approx 0 \mathrm{~mm}$, the nose (Stesalite end disc) of the inner field cage is at about $z \approx 250 \mathrm{~mm}$, and the total drift region is $\approx 1500 \mathrm{~mm}$ long (hence ending at $z \approx 1000 \mathrm{~mm}$ ). Those numbers imply that, in a spill of about $400 \mathrm{~ms}$, the ion cloud just reaches the $z$ position of the target.

The constant increase of distortions during the spill is easily explained: the drift electrons generated by tracks of triggered events have to cross an increasing number of ions produced by the beam, as the thickness of the ion cloud to be traversed increases from 0 to about $600 \mathrm{~mm}$ from the start to the end of a $400 \mathrm{~ms}$ long beam spill. Before the period of linear increase in strength of the distortions, one expects a short period of zero distortions: the ion cloud produced in the amplification region first has to reach the drift volume. This period is estimated to be about $25 \mathrm{~ms}$. Between the period of zero effect and linear growth one expects a smooth transition given by the ion diffusion and the difference in length of the drift path in the regions around the anode and gating grid wires.

These expectations can be tested by comparing the distortions affecting tracks generated at different values of $z$ in the TPC. Tracks within a limited angular range approximately perpendicular to the beam direction are used for this analysis since their trajectory lies within a small range in $z$. (Tracks between \pm 30 degrees with respect to the normal are accepted.) If the dynamics of the ion cloud is correctly described with the considerations given above, tracks generated in the Stesalite end disc of the IFC (large positive $z$ ) and in the target should be affected by the same distortions at any time of the spill, because their drift electrons have to cross the same ion cloud. Fig. 5 demonstrates that the distortions observed for these two groups of tracks are indeed identical.

On the other hand, tracks generated in a long target (e.g. a two interaction length long 
aluminum target of $\approx 80 \mathrm{~cm}$ ) at negative $z$ values in the TPC (half-way between the pad plane and $z=0$ ), should show a saturation of the distortions before the end of spill. Figure 6 indeed shows that the distortion of tracks produced at $z \approx-250 \mathrm{~mm}$ is no longer increasing after about $130 \mathrm{~ms}$, consistent with the predictions given in Section 5 . The ion wavefront is expected to reach $z \approx-250 \mathrm{~mm}$ after about $125 \mathrm{~ms}$, and the thickness of ion cloud to be traversed by the drift electrons remains constant. (Given the rather uniform beam intensity during the spill, per unit time the same number of ions are produced at the pad plane as the number which cross the ideal $z=-250 \mathrm{~mm}$ plane.) Fig. 6 also shows that tracks produced at increasingly larger $z$ exhibit the distortion saturation at increasingly later times.

Ions are no longer generated in the amplification region after the end of the beam spill. Thus it is expected that the ion cloud remains of constant thickness (about $600 \mathrm{~mm}$ ) between spills, and that it drifts into the direction of positive $z$, gradually freeing the active volume from distortions starting first with the negative $z$ region of the TPC. Cosmic-ray tracks recorded for calibration taken during the time between spills allow this behaviour to be studied. To be able to study the distortion effects, tracks are selected which approximately cross the IFC region. The distortions in the measurements of the trajectory on either side of the region of the IFC are of opposite sign (if expressed in Cartesian coordinates). As a measure of the distortions the variable $\Delta \phi^{0}$ is defined as the difference of the measured $\phi$ of the top half of the track compared to the $\phi$ measured for the complete cosmic-ray track. Figure 7 shows the $\Delta \phi^{0}$ for two time periods with different delays from the end of the preceding spill. Indeed the two back-to-back segments of cosmicray tracks taken at negative $z$ become progressively less affected by distortions, and the distortion-free region expands with time (while the ions drift towards more positive $z$ ).

Subtle effects can be shown and explained by analyzing the cosmic-rays taken between spills. From Fig. 8 it can be observed that tracks at very large positive $z \geq 550 \mathrm{~mm}$ see a different distortion strength at the beginning of the inter-spill (first cosmic) with respect to the distortion seen after about additional $80 \mathrm{~ms}-100 \mathrm{~ms}$ after the end of the preceding spill. This occurs despite the fact that in both cases their drift electrons cross the same ion cloud thickness. The front of the cloud has meanwhile just moved by less than $200 \mathrm{~mm}$, and did not reach yet $z=500 \mathrm{~mm}$. This holds true even if taking into account that the trigger system was programmed to provide a wait-state of about $140 \mathrm{~ms}$ between the end-of-spill and the first inter-spill cosmic. The reasons for this behaviour can be fully understood: during the $80 \mathrm{~ms}-100 \mathrm{~ms}$ difference between the first and last inter-spill cosmic-rays, a fraction of the ion cloud has passed the position of the inner fieldcage and disc, entering a region where the electrostatic configuration of the field-cage is completely different. In the region where the electric field is only formed by the cylinder of the outer field-cage, the distortion field produced by the ions in the disc with $R$ smaller than the drifting electron clusters is only attractive towards the origin: the repulsion term of the inner field cage is missing, and the outward component of the ions at radii larger than the position of each drift electron is null. Therefore, there is a range in $z$ in which the the drift electrons feel an inward force, thus partially compensating the usual distortion at small $\mathrm{R}$ in the $z$ range, where both the ions and the inner field cage are present. This is why the cosmic-ray tracks crossing the TPC at large $z$ values are more distorted at times directly after the spill compared to later times. There is a $z$ value (taking in account the trigger shift), corresponding to the end/cap of inner field cage, where the distortions have 
a maximum, see Fig. 8.

\section{Experimental determination of the $R \phi$ distortion using elastic scattering}

When trying to measure the effect of distortion in an unbiased way it is important to avoid the use of reference quantities which can themselves be affected by the distortions. For example, a biased result is obtained if one measures first the track curvature using the distorted trajectory and if one then at a later stage uses this curvature in combination with fixed references such as vertex position and hits of RPCs to predict the undistorted position of charge clusters inside the gas volume. This approach was suggested in (18) and we show in the following that such a procedure can be avoided.

On the contrary, as already discussed in Sec. 4, elastic scattering off $\mathrm{H}_{2}$ can be used to predict the complete undistorted trajectory without making use of quantities which are affected in any way by the distortions. By measuring the scattering angle $(\theta)$ of the forward going particle with respect the direction of the beam particle (whose momentum is precisely selected by the beam setting) the four-momentum of the proton recoiling at large angle is derived from the elastic scattering kinematics. This provides a reference quantity suitable to actually measure the distortion. The knowledge of the four-momentum of the large-angle proton is the key to extend the method to directly determine the $R \phi$ displacement of the clusters. This approach avoids completely the introduction of dependencies on parameters affected by the distortions.

The full trajectory of the large-angle proton in the active region of the TPC is calculated by using the geometry of the detector as described in detail in the simulation program. The simulation program takes into account all the details of the materials traversed by the scattered proton. This creates for every individual pad row an unbiased reference sample as function of $N_{\text {evt }}$ free from a-priori assumptions.

The procedure was applied to the five reference hydrogen data sets available: $3 \mathrm{GeV} / c$, $5 \mathrm{GeV} / c$ and $8 \mathrm{GeV} / c$ with a $60 \mathrm{~mm}$ long target and $3 \mathrm{GeV} / c$ and $8 \mathrm{GeV} / c$ with a $180 \mathrm{~mm}$ long target. The average difference (along $r \phi$ ) of the position of the predicted trajectory and the measured $r \phi$ coordinate are shown in Fig. 9 as a function of $N_{\text {evt }}$ for data taken with the $180 \mathrm{~mm}$ hydrogen target in the $3 \mathrm{GeV} / \mathrm{c}$ beam. For each pad plane row a straight line fit of the distortion measurements during the whole spill is made. The slope of the best straight line fit is used as monitor of the growth of the distortion versus time, is called distortion strength, and is given in units of growth of the distortion per recorded event. Figure 10 shows the results obtained for the $3 \mathrm{GeV} / c$ beam impinging on the $180 \mathrm{~mm}$ target and the $5 \mathrm{GeV} / c$ data taken with the $60 \mathrm{~mm} \mathrm{H}_{2}$ target. The distortion strength increases during the whole spill, consistent with the behaviour of $d_{0}^{\prime}$ shown in fig. 5. Most interestingly, Fig. 10 also shows that the direction of the distortion changes sign from the inner TPC rows to the outer ones, and that there is a cross-over point of vanishing distortion. The change of sign can be explained qualitatively with electrostatic arguments taking into account the fact that the HV power supply keeps the inner and outer field cages at a constant voltage. These arguments will be worked out in detail below. One can further observe that the absolute value of the outward field component at row number one is larger than the absolute value of the inward field component at row 
number twenty.

\section{Phenomenological Model}

\subsection{Simple discrete model}

A phenomenological model can be constructed based on the fact that the field which is responsible for the force acting on each drift electron is equivalent to a vector sum of two field systems:

- a field system where ions, in a given angular section at $R$ values internal to the drift electron position contribute to attract the drift electrons inward;

- a field system where ions, in a given angular section at $R$ values external to the drift electron position, contribute to attract the drift electrons outward.

In this description, the fixed voltage of the inner field cage plays a crucial role: it breaks the circular symmetry by shielding the charges in its shadow. This model makes it possible to understand all the peculiar features of the TPC dynamic distortions:

A simple, approximate algorithm can be written, using a discrete representation at the row-level. Despite of its simplicity such a model can predict the basic features with surprising accuracy. Various models of the distribution of the density of ions as a function of $R$ and of the $\mathrm{R}$ dependence of the electric field can be readily modelled. These models predict the change of sign of the distortion strength between the first and last pad rows, as well as the position of the pad row with vanishing distortion. The system can be modelled with only a very small number of fixed geometrical parameters of the TPC and a free parameter describing the overall distortion strength. A comparison with the distortion measured with the data taken with the $180 \mathrm{~mm}$ hydrogen target exposed to the $3 \mathrm{GeV} / c$ beam is shown in Fig. 11.

\subsection{General Analytical Solution}

The radial electric field distorting the trajectory of the drift electron is due to two components. Given any radius $r_{e}$ where a drift electron is supposed to travel parallel to $z$ in the TPC, a distortion term is directly due to the contribution of the positive ion cloud integrated from the first row up to $r_{e}$. The cylindrical shells of the ion cloud, external to $r_{e}$, do not contribute because of the Gauss theorem. A second distortion term is due to the induced excess of negative charge onto the conducting surface of the inner field cage. Such a field is equivalent to the one of a charged wire along the $z$ axis.

The two distortion terms have opposite sign. The repulsive (outward force) term prevails at small $R$. The attractive (inward force, same sign as the static distortion force) term prevails at large $R$. There must be a radial position with vanishing distortion effect. A simple discretization of the field due to the ions alone, at the radius $R=r_{e}$, can be written 
as:

$$
E_{r}^{1}(R)=\frac{2 \sum_{i} Q\left(R_{i}\right)}{Z R}, \quad R_{i}<R
$$

because the field due to each discrete cylindrical shell is equivalent to the one generated by a wire length $Z$ charged with the charge $Q\left(R_{i}\right)$ contained in the volume of each shell. $\mathrm{R}$ is the radius at which the field has to be computed, which is at the same distance from all effective wires along z generated by each cylindrical shell, according to the Gauss theorem with the assumption of uniform charge density along $z$ and over the cylindrical angle.

The ions are generated by the amplification of all the ionization electrons produced by all the charged particles that traverse the TPC during the spill. The ion cloud in the drift volume is populated by ions which cross the grid in front of the anode wires either because the grid was open or not perfectly closed. Interactions of beam and halo particles with the target and its surrounding materials produce secondary particles most of each reach the outer field cage of the TPC. Therefore, a $1 / r$ dependence of the ion charge distribution in space is a very good initial estimate. This consideration gives:

$$
E_{r}^{1}(R)=\frac{K_{1}}{R} \int_{R_{1}}^{R} \frac{d r}{r+c},
$$

where $K_{1}$ is a charge normalization factor depending on the beam and target setting, $c$ is the parameter fixing the charge at any given radius (also depending on beam and target setting), $R_{1}$ is the internal radius of the ion cloud (close to the first pad row).

On the other hand, the field due to the induced negative charge $-Q_{2}$ on the inner field cage is given by:

$$
E_{r}^{2}(R)=-\frac{K_{2} Q_{2}}{R}
$$

This relation is correct as long the inner field cage is surrounded by the ion cloud, as it is indeed the case, because the ion velocity is such that the ion wavefront reaches the target position $z=0$ by the end of the spill. Thus the total radial distortion field can be expressed as:

$$
E_{r}(R)=E_{r}^{1}+E_{r}^{2}=\frac{1}{R}\left(K_{1} \ln \left(\frac{R+c}{R_{1}+c}\right)-K_{2} Q_{2}\right)
$$

The general expression can finally be written as:

$$
E_{r}(R)=\frac{K}{R}(\ln (R+c)-y)
$$

where $K$ and $y$ absorb the various constant factors. This equation contains the setting dependencies of the charge distribution and beam intensity in the form of the free parameters $K, y$ and $c$ and they can be used to parametrize and correct the dynamic distortions 
of any setting. The number of parameters can be reduced by the condition:

$$
\int_{R_{1}}^{R_{2}} E_{r}(R) d r=0
$$

where $R_{1}$ is the conducting surface of the inner field cage and $R_{2}$ is the conducting surface of the outer field cage. This condition uses a natural assumption that the power supply manages to keep the voltage at the nominal value at each resistor partition (we assume here that the static distortions have been corrected).

The absolute scale to be used in Eq. 5 can be fixed with the help of experimental data. In the hydrogen settings, the direct measurement of the position of the clusters can be used, while in the data taken with the other targets the time-dependence of the distribution of $\left\langle d_{0}^{\prime}\right\rangle$ is a good estimator.

Assuming a uniform distribution of the ion charge in the volume, Eq. 6 can be resolved analytically if one requires $c \ll R$ (justified by the charge generation mechanism around the beam), and if one neglects the difference between inner field cage radius and the inner radius of the ion cloud (which is in any case internal to the first pad ring radius) Then this gives a solution for the value of $y$ :

$$
y=\ln \left(R_{0}\right) \quad \text { where } R_{0}=\sqrt{R_{1} R_{2}}
$$

i.e. $R_{0}$ is the geometric average of the radial positions of the conducting surfaces and is the radius for which the resulting field is zero. Thus this could be predicted from first principles.

A detailed comparison with the measurements is shown in Fig. 11, using Eq. 5 and 7 and the geometric dimensions of the TPC surfaces and pad size. This shows a remarkable agreement between the distortion data for the $3 \mathrm{GeV} / c$ hydrogen data and the prediction based on the uniform charge distribution. If one uses a non-uniform space charge distribution the numerical solution of Eq. 6 gives similar results.

Both with the numerical integration code and with the analytical calculation, the electrostatic problem has been solved also for different density distributions (and different $R$-dependencies of the electric field taking into account more or less strong edge effects). This may be useful in case of particular beam settings. For example, below is given the formula expressing the radial electric field for the case of a $1 / R$ superficial density distribution on each cylindrical shell (i.e. constant charge in the volume of each cylindrical shell):

$$
E_{r}(R)=E_{0}\left(1-\frac{R_{2}-R_{1}}{R \ln \left(R / R_{1}\right)}\right) .
$$

We have shown that the method works for other density distributions; however the density distribution used in the paper is the one resulting from the raw measurements of the pad occupancies. 


\section{Correction method}

With the models described above and the direct measurements a distortion strength as a function of row number is determined. The strength is measured as a residual, therefore it can be used as the basis for an $R \phi$ correction applied to clusters measured on tracks. However, before the correction can be used for each target and beam setting where the elastic scattering cannot be measured, one has to correlate the characterization with the behaviour of one or more global track parameters. The analysis of the elastic scattering data shows that the largest effect of the distortions is seen in the pad rows nearest to the centre. Therefore, one of the best candidates in this respect is $d_{0}^{\prime}$ which is easy to measure for each track and therefore gives statistically significant reasults for each data set.

The behaviour of $d_{0}^{\prime}$ as a function of the event-in-spill shows a first part with, essentially, no distortion, then a quadratic rise, followed by a linear behaviour until an upper limit is reached. Empirically, this behaviour can be understood from the previously described ion cloud dynamics. At the beginning of the spill the TPC is distortion-free; soon after the onset of the distortion the effect stabilizes into a linear increase until levelling-off at the point in time where all the distance travelled by the ionization charges is filled by the ion cloud. The saturation is in practice not reached for tracks emanating from the target during the spill. The intermediate region approxamated by the quadratic rise is understood as the onset of the effect when the front of the ion cloud enters in the drift region. Due to the different paths the ions travel in the amplification region, the front of the cloud is not sharp. The behaviour is seen in Fig. 12 (left panel). It is observed that

the dependence of $d_{0}^{\prime}$ as a function of $N_{\text {evt }}$ shows the three regimes of the distortion as described above. Three calibration parameters are extracted with an iterative procedure: the value of $N_{\text {evt }}$ up to which there is no distortion, the value of $N_{\text {evt }}$ where the rise changes from quadratic to linear, and an overall scale factor. A time-dependent upper limit to the growth is also defined to take into account the fact that the distortion saturates at a different value of $N_{\text {evt }}$ depending on the $z$ position of the original ionization charge. This is not a free parameter.

To take into account the different characteristics of the initial charge distribution the data taken with the $3 \mathrm{GeV} / c$ beam use the corrections determined using the $3 \mathrm{GeV} / c$ hydrogen data, the $5 \mathrm{GeV} / c$ beam corrections use the $5 \mathrm{GeV} / c$ hydrogen data, while the $8 \mathrm{GeV} / c$ hydrogen data are used for the higher momenta.

For a given setting all data are first reconstructed without any correction for dynamic distortions and with a default (setting-independent) correction for static distortions. The characteristics of the dependence of $d_{0}^{\prime}$ on $N_{\text {evt }}$ is then used to determine the initial values for the four parameters (three for the dynamic distortions and one for the static correction.) The row-by-row dependence is characterized by a set of 20 numbers (one of three sets as explained above). Then this set is multiplied by a single strength factor, depending on the value of $N_{\text {evt }}$. As only additional complication, the strength factor has a $z$ and $R$ dependent ceiling to take into account the saturation.

The iterative procedure is terminated if the $d_{0}^{\prime}$ curves of positive and negative pions are equal within $\pm 2 \mathrm{~mm}$ over the whole spill. Typically, only one extra iteration is needed to obtain the required precision. This indicates that the characteristics of the $d_{0}^{\prime}$ distributions 
describe the overall distortion strength reliably. The result of the procedure is shown in Fig. 12. The small difference between the positive and negative pions around $N_{\text {evt }}=50$ has no effect on the measurement of the momentum, but shows that the simple parabolic model describing the period of gradual onset of the distortions is not completely accurate. The approximations used in the method are valid for values of $\left\langle d_{0}^{\prime}\right\rangle$ not exceeding $20 \mathrm{~mm}$. The shape of the $\left\langle d_{0}^{\prime}\right\rangle$ distribution as a function of $N_{\text {evt }}$ shows clearly up to which value of event-in-spill the fitted parameters can be used. This maximum value is setting dependent, and is larger for beam settings which were better focused, for beams tuned at lower intensity and for targets of lower $z$. The target material dependence is introduced by the multiplicity of the interaction products, which is higher for higher $z$. In practice, this criterion does not represent a significant loss in final statistics of the data sets. On average more the $80 \%$ of the data can be reliably corrected. The data sets which have had to be truncated most turn out to be the ones which were not statistics limited in any way (e.g. the high $z$ data sets).

\section{Performance of TPC after correction}

In order to check the results of the corrections for the distortions effects a number of control distributions were evaluated for each analysed data-set.

One control plot is the overall $p_{\mathrm{T}}$ distribution of all tracks as a function of $N_{\text {evt }}$. Figure 13 shows the distribution in $Q / p_{\mathrm{T}}$, where $Q$ is the measured charge of the particle, for six groups of tracks, each corresponding $50 n<N_{\text {evt }} \leq 50(n+1)$ (for $n$ ranging from zero to five). The distributions have been normalized to an equal number of incident beam particles, with the first group as reference. In the left panel, no dynamic distortion correction have been applied and a clear difference of the distributions is visible. One should note that the momentum measurement as well as the efficiency is modified. The right panel shows the distributions after the corrections. The distributions are no longer distinguishable. To understand the asymmetry of positively and negatively charged tracks, one should keep in mind that no particle identification was performed. Thus both protons and pions contribute to the positives while the $\pi^{-}$'s are the only component of the negative particles.

A more direct test of the effect of the correction on the measurement of momentum is shown in Fig. 14. Four groups of tracks were selected, two classes of proton tracks and pions separated on the basis of their charge. A sample of relatively high momentum protons was selected using their range to set a lower limit. The protons were required to produce a hit in two RPC layers. A fixed window with relatively high values of $\mathrm{d} E / \mathrm{d} x$ in the TPC ensured the particle identification as protons and limited the maximum momentum. Another window with higher values of $\mathrm{d} E / \mathrm{d} x$ selects protons with a lower momentum. The pions are selected again by $\mathrm{d} E / \mathrm{d} x$, which is only possible for low momentum values (around $100 \mathrm{MeV}$ ). Positively charged and negatively charged pions are treated separately. The angle of the particles is restricted in a range with $\sin \theta \approx 0.9$, ensuring a small range of $p_{\mathrm{T}}$. In the left panel (uncorrected data) one observes a variation of $\approx 5 \%$ for the high $p_{\mathrm{T}}$ samples. The corrected data stay stable well within $3 \%$. The low $p_{\mathrm{T}}$ pion data remain stable with or without correction. The width of the measured momentum distributions 
remains the same over the length of the spill, indicating that also the resolution is well corrected. It should also be noted that there is an effect on the efficiency. While the efficiency to find a collection of clusters as a track is not modified by the distortions, the requirement that the track is pointing to the target does introduce an efficiency loss for the uncorrected data. This loss is visible as an increase in the error bars on the measurements.

From the combination of the two sets of control plots one can conclude that the dynamic distortion corrections achieve a uniform efficiency and a constant measurement of momentum over the whole spill. Since the initial characterization of the TPC performance and calibration was determined using the first part of the spill which is not affected by dynamic distortions one expects that the calibration remains applicable. The systematic errors on these quantities remain approximately equal: although an additional correction would improve the situation, more events are now used with larger corrections applied to their tracks.

\section{Results for $8.9 \mathrm{GeV} / c$ Be data}

Finally, a comparison of the end-product of the analysis, double-differential cross-sections, before and after the corrections can be made.

The measured double-differential cross-sections for the production of $\pi^{+}$and $\pi^{-}$in the laboratory system as a function of the momentum and the polar angle for each incident beam momentum were measured for many targets and beam momenta. These results are in agreement with what previously found using only the first part of the spill and using no dynamic distortions corrections. Of course, both analyses only use the data for which their calibrations are applicable. Thus a lower statistics sample is used for the uncorrected data. Making this comparison using the $8.9 \mathrm{GeV} / c$ Be data has the advantage of using the data set with the highest statistics, thus achieving the best possible comparison. Figures 15 and 16 show the ratio of the cross sections without and with the correction factor for dynamic distortions in $8.9 \mathrm{GeV} / c$ beryllium data. The error band in the ratio takes into account the usual estimate of momentum error and the error on efficiency, the other errors are almost fully correlated. The agreement is within $1 \sigma$ for most of the points, confirming the estimate of differential systematic error. The statistical error bar represents the statistics of the non-overlapping events.

\section{Conclusions}

The HARP TPC suffers a rather large number of operational problems. The dynamic distortions observed for the particle trajectories were tackled after the other problems had been corrected. The overall characteristics of the effect of these distortions were described. Mainly the measurement of curvature and the extrapolation to the target were affected. It was shown that the origin of the distortions is fully understood both theo-

retically and experimentally. An experimental method to obtain a direct measurement of the distortions on the trajectory in space was developed. The $d_{0}^{\prime}$ variable has been identified to be a sensitive indicator of dynamic distortions both with $\mathrm{H}_{2}$ targets and heavier 
targets. The effect of dynamic distortions on the particle trajectories in the TPC has been measured directly with $\mathrm{H}_{2}$ targets by exploiting the forward spectrometer and the kinematics of elastic scattering. A simple model of the generation of dynamic distortions and a correction algorithm which depends on parameters that are controlled by the $d_{0}^{\prime}$ variable were developed. By monitoring the distortion strength with the $d_{0}^{\prime}$ observable the correction algorithm can be applied to all data sets taken with different targets. The TPC performance (momentum scale and absolute efficiency) were measured during the full spill by using data with hydrogen targets. The results of the corrections show that the performance of the TPC is restored for the vast majority of the data.

\section{Acknowledgments}

We gratefully acknowledge the help and support of the PS beam staff and of the numerous technical collaborators who contributed to the detector design, construction, commissioning and operation. This work would not have been possible without the combined use of all the detector components of HARP. We would like to thank our colleagues from the HARP collaboration for their contribution to this work.

The experiment was made possible by grants from the Institut Interuniversitaire des Sciences Nucléaires, Ministerio de Educacion y Ciencia, Grant FPA2003-06921-c02-02 and Generalitat Valenciana, grant GV00-054-1, CERN (Geneva, Switzerland), the German Bundesministerium für Bildung und Forschung (Germany), the Istituto Nazionale di Fisica Nucleare (Italy), INR RAS (Moscow), the Particle Physics and Astronomy Research Council (UK) and the Swiss National Science Foundation, in the framework of the SCOPES programme. We gratefully acknowledge their support.

\section{References}

[1] M.G. Catanesi et al., HARP Collaboration, "Proposal to study hadron production for the neutrino factory and for the atmospheric neutrino flux", CERN-SPSC/99-35 (1999).

[2] M.G. Catanesi et al., HARP Collaboration, "The HARP detector at the CERN PS", Nucl. Instrum. Meth. Phys. Res. A, A571 (2007) 527; A571 (2007) 564.

[3] M. G. Catanesi et al. [HARP Collaboration], "Measurement of the production of charged pions by protons on a tantalum target", Eur. Phys. J. C 51 (2007) 787, arXiv:0706.1600.

[4] M.G. Catanesi et al., HARP Collaboration, "Large-angle production of charged pions by $3 \mathrm{GeV} / c 12 \mathrm{GeV} / c$ protons on carbon, copper and tin targets", Eur.Phys.J.C53 (2008) 177, arXiv:0709.3464 [hep-ex].

[5] M.G. Catanesi et al., HARP Collaboration, "Large-angle production of charged pions by $3 \mathrm{GeV} / c 12.9 \mathrm{GeV} / c$ protons on beryllium, aluminium and lead targets", Eur. Phys. J. C 54 (2008) 37, arXiv:0709.3458 [hep-ex].

[6] M.G. Catanesi et al., HARP Collaboration, "Large-angle production of charged pions with 3-12.9 GeV/c incident protons on nuclear targets", Phys. Rev. C 77 (2008) 055207, arXiv:0805.2871 [hep-ex]. 
[7] S.R. Amendolia et al., "TPC90, a test model for the ALEPH time projection chamber", Nucl. Instr. and Meth. A252 (1986) 392.

[8] J.W.E. Uiterwijk, J. Panman and B. Van de Vyver, "A C++ object-oriented toolkit for track finding with k-dimensional hits", Nucl. Instrum. Methods A560 (2006) 317.

[9] J. Knobloch et al., "Status of the Reconstruction Algorithms for Aleph", ALEPHNote 88-46.

[10] M.C. Morone, "Evaluation of Silicon sensors for the ATLAS Silicon Tracker, and TPC Reconstruction in the HARP Experiment", Ph.D. Thesis, University of Geneva, 2003.

[11] Silvia Borghi, Ph.D. Thesis, "Hadron production cross section measurement with the HARP large angle detectors", CERN-THESIS-2007-034, University of Geneva, 2006.

[12] N.I. Chernov, G.A. Ososkov, "Effective algorithms for circle fitting", Computer Physics Communications 33 (1984) 329.

[13] L. Howlett, "Simulation and Correction of Cross Talk in the HARP Time Projection Chamber", Ph.D. Thesis, Sheffield, 2004.

[14] G. Van Buren et al., "Correcting for distortions due to ionization in the STAR TPC", Nucl. Instrum. Methods A566 (2006) 22.

[15] P. Temnikov et al., "pp Elastic Scattering at $3 \mathrm{GeV} / c$ with Liquid $\mathrm{H}_{2}$ Targets in HARP", INFN-LNL (REP) 209-06 and HARP-Note 06-003.

[16] M.G. Catanesi et al., HARP Collaboration, "Absolute momentum calibration of the HARP TPC", J. Inst. 3 (2008) P04007.

[17] G. Dellacasa et al., ALICE Collaboration, "ALICE Time Projection Chamber: Technical Design Report", CERN-LHCC-2000-001, ALICE TDR-7.

[18] V. Ammosov et al., "The HARP Time Projection Chamber: Characteristics and Physics Performance", Nucl. Instrum. Methods A588 (2008) 294. 

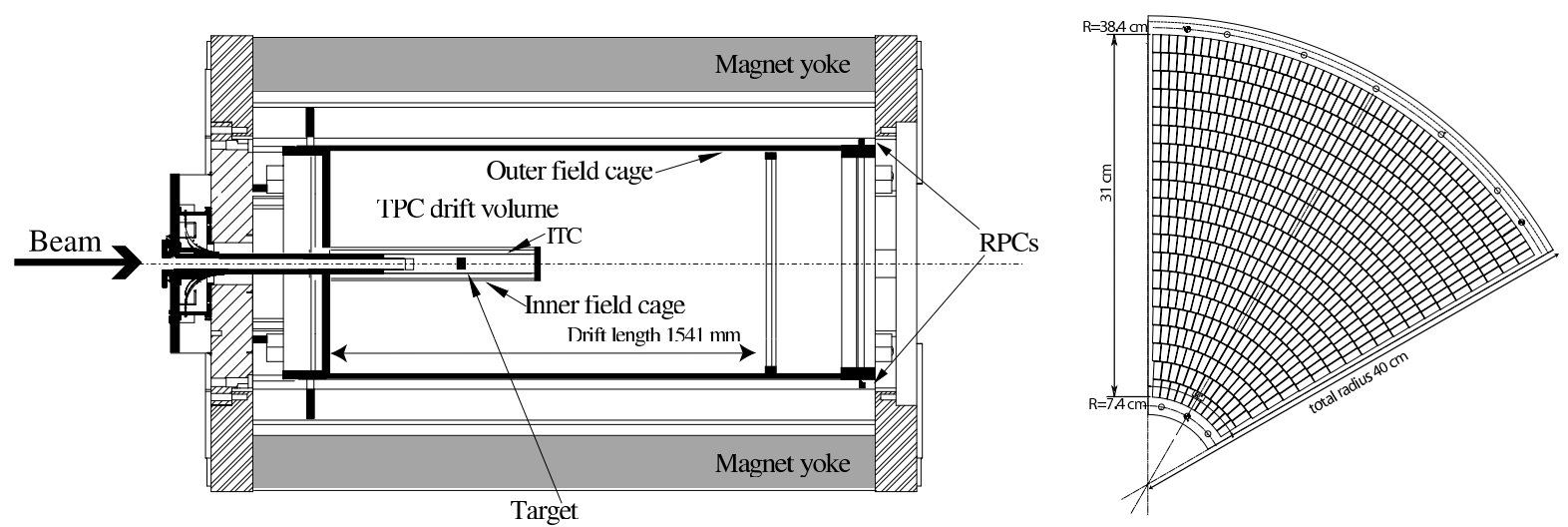

Fig. 1. Schematic layout of the TPC. The beam enters from the left. Starting from the outside, first the return yoke of the magnet is seen, closed with an end-cap at the upstream end, and open at the downstream end. The inner field cage is visible as a short cylinder entering from the left. The ITC trigger counter and the target holder are inserted in the inner field cage. The RPCs (not drawn) are positioned between the outer field cage and the coil. The drift length is delimited on the right by the cathode plane and on the left by the anode wire planes. On the right the mechanical drawing of a sector of the TPC, the layout of the pads is indicated.
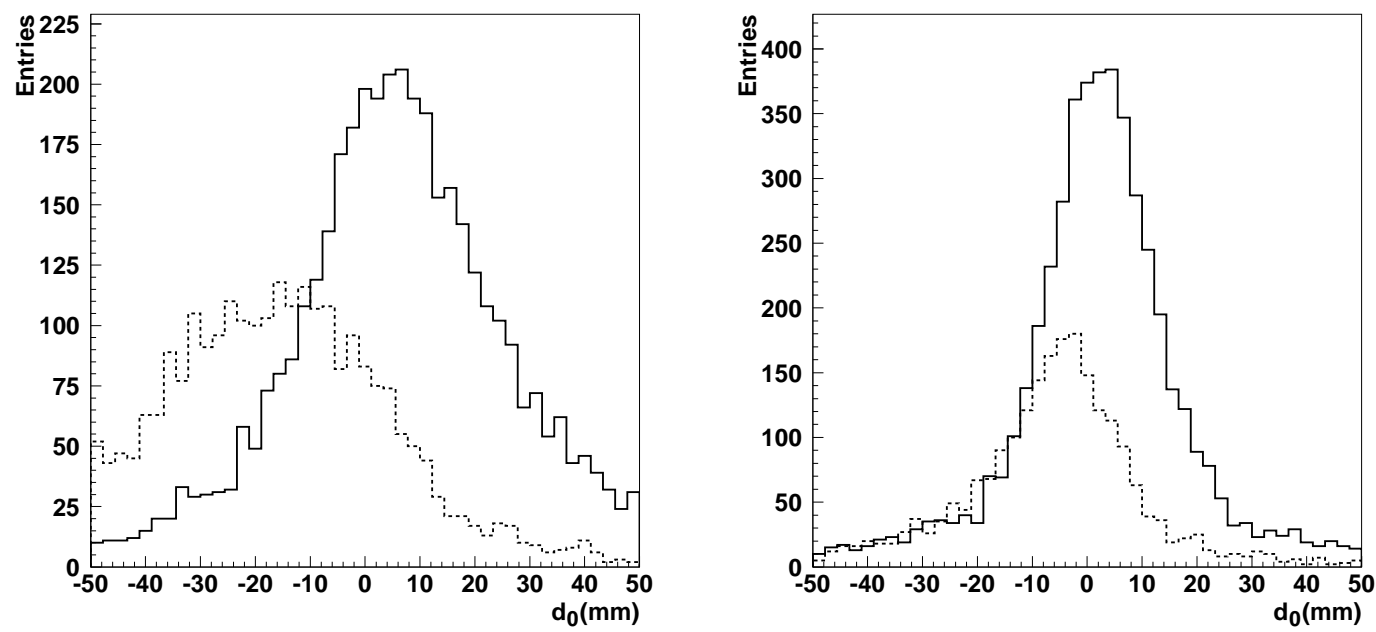

Fig. 2. The $d_{0}$ distributions in a $8 \mathrm{GeV} / c$ beam exposure on a $5 \% \lambda_{\mathrm{I}}$ Be target. Left panel: run 9540. Right panel: run 9455. The histograms drawn with continuous lines are for positive particles and the the ones with dotted lines for negative particles. The two runs are taken close to each other in time, one just before and the other after re-tuning of the beam. 


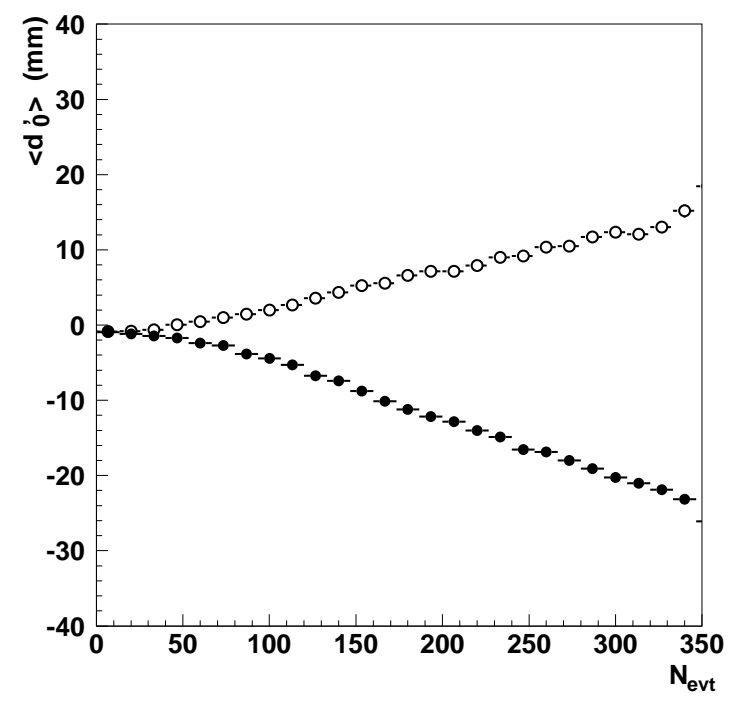

Fig. 3. Example of monitoring of the dependence of the distortion on the time during the spill. $d_{0}^{\prime}$ as function of the event number in the spill for a $3 \mathrm{GeV} / c$ beam exposure on a Ta target. Open circles: positively charged particles; closed circles: negatively charged particles.
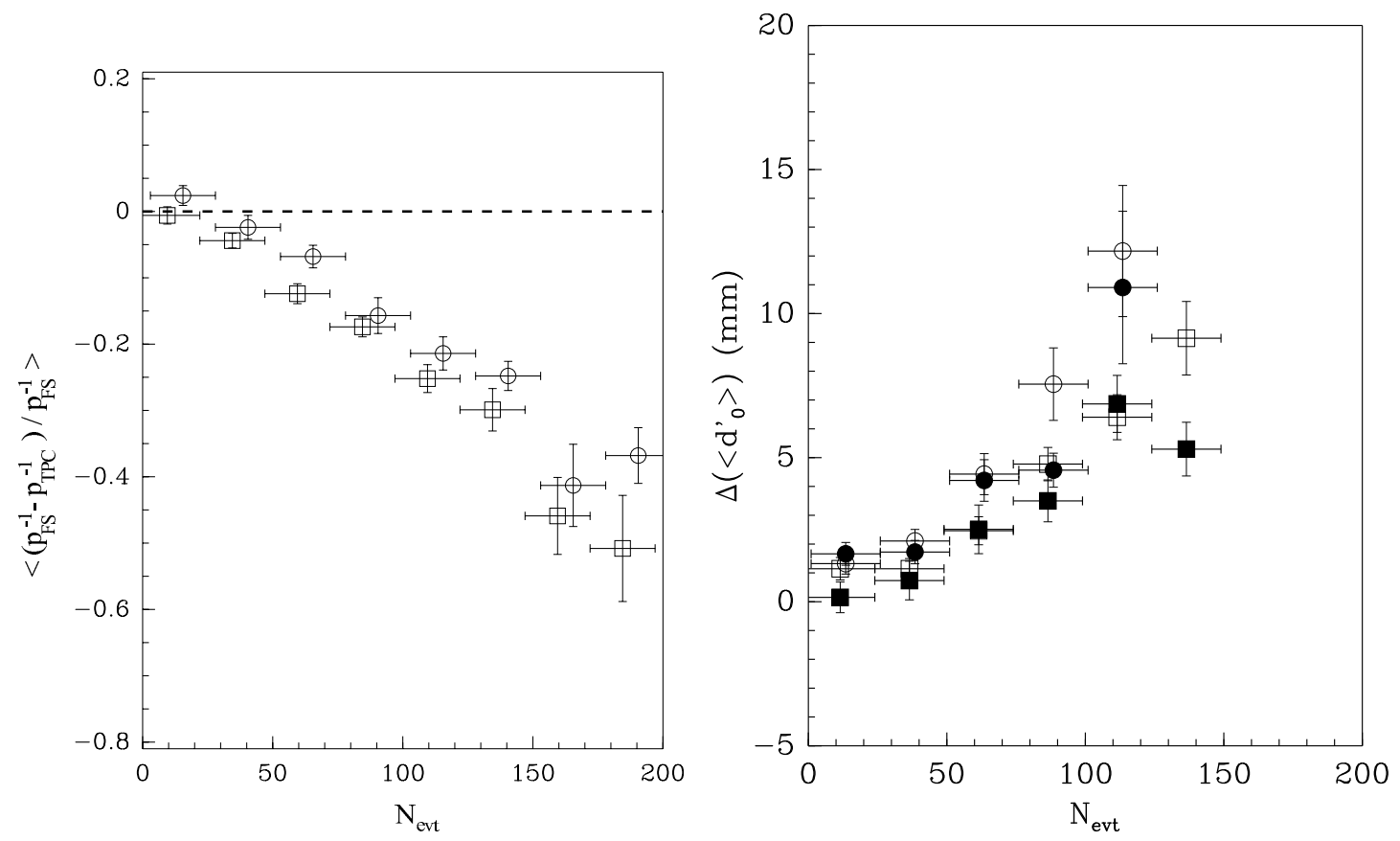

Fig. 4. Left panel: The shift in average momentum for elastic scattering data $(3 \mathrm{GeV} / c$ : open squares, $5 \mathrm{GeV} / c$ : open circles) measured with elastic events as a function of the momentum predicted by the forward scattered track. It is as function of the event number in spill for different predicted momentum. The momentum estimator from the fit not constrained by the impact point of the incoming beam particle is used here. Right panel: The shift in average $d_{0}^{\prime}$ as a function of the event number in spill for elastic scattering data $(3 \mathrm{GeV} / c$ : filled and open boxes, $5 \mathrm{GeV} / c$ : filled and open circles) measured with elastic events as a function of the momentum predicted by the forward scattered track. The open symbols show the data for momenta below $450 \mathrm{MeV} / c$ and the filled symbols for momenta above $450 \mathrm{MeV} / c$. 

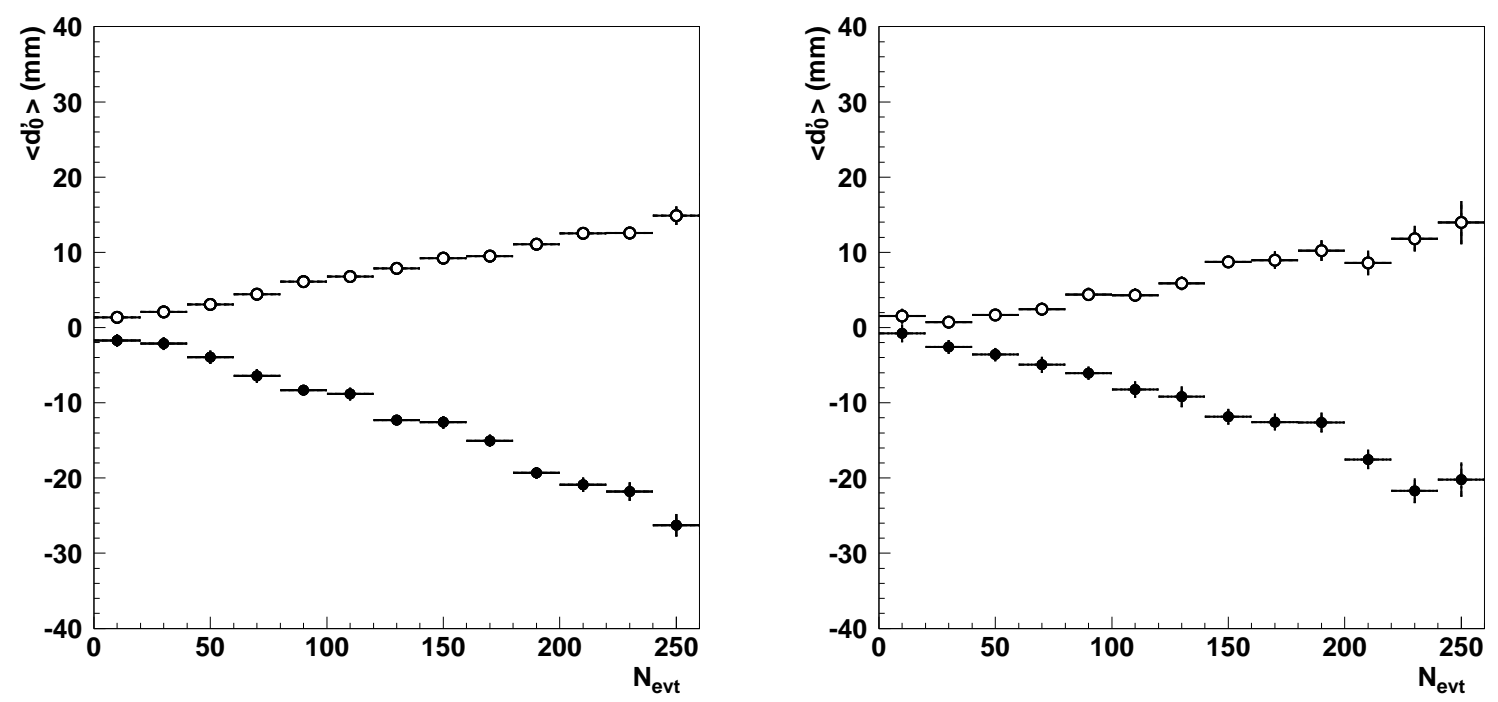

Fig. 5. The average $d_{0}^{\prime}$ as a function of event number in spill for positively (open dots) and negatively (filled dots) charged particles for the hydrogen target for $3 \mathrm{GeV} / c$ beam. Left panel: distortion of tracks originated in the target $(z \sim 0 \mathrm{~mm})$. Right panel: distortion of tracks generated in the Stesalite $(z \sim 268.5 \mathrm{~mm})$. Open circles: positively charged particles; closed circles: negatively charged particles.

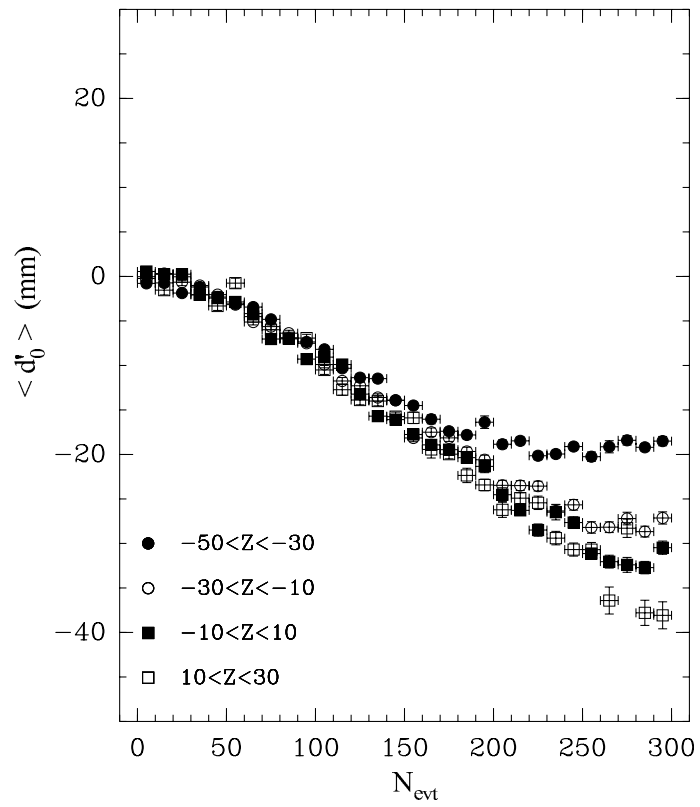

Fig. 6. Average $d_{0}^{\prime}$ measured as a function of the event number in spill using a long aluminium target. The data are divided into four regions of the $z$ of the interaction point. $z_{0}$ : $-50 \mathrm{~cm} \leq z<-30 \mathrm{~cm}$ (closed circles); $z_{1}:-30 \mathrm{~cm} \leq z<-10 \mathrm{~cm}$ (open circles); $z_{2}$ : $-10 \mathrm{~cm} \leq z<+10 \mathrm{~cm}$ (closed squares); $z_{3}:+10 \mathrm{~cm} \leq z<+30 \mathrm{~cm}$ (open squares). The data are shown for $\pi^{-}$tracks. The event number in spill (in first approximation corresponding to time) where the deviation of the average of the different series of points saturate clearly show the ion mobility. During the first $\approx 25$ events $(30 \mathrm{~ms}$ ) no deviation is visible, consistent with the fact that the ions - created in the amplification region - have not yet reached the drift volume. During the following $\approx 50$ events the derivative of the slope increases, showing the ion diffusion. 


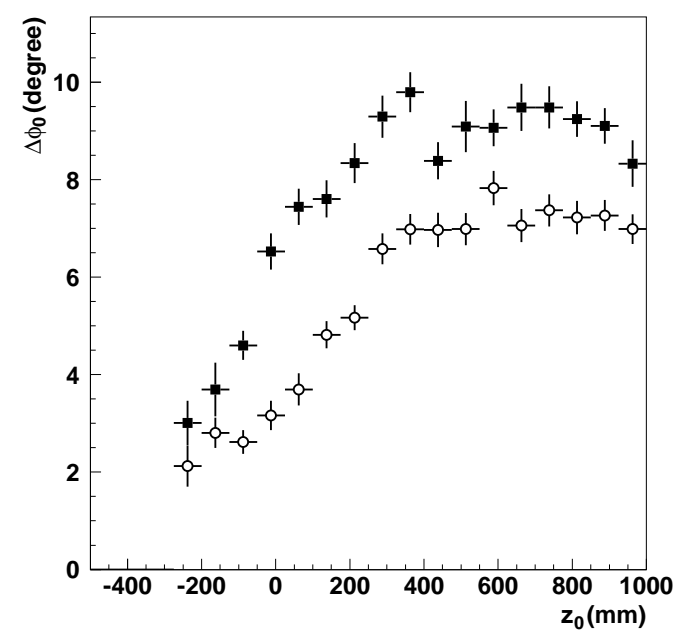

Fig. 7. The average $\Delta \phi_{0}$ for two time periods with different delays from the end of the preceding spill. Cosmic rays taken within the first $175 \mathrm{~ms}$ after the end of the spill are shown with closed circles, while the tracks taken between $250 \mathrm{~ms}$ and $300 \mathrm{~ms}$ after the end of the spill are shown with open circles. The distortions tend to zero at $z$ values which are already passed by the ion packet.

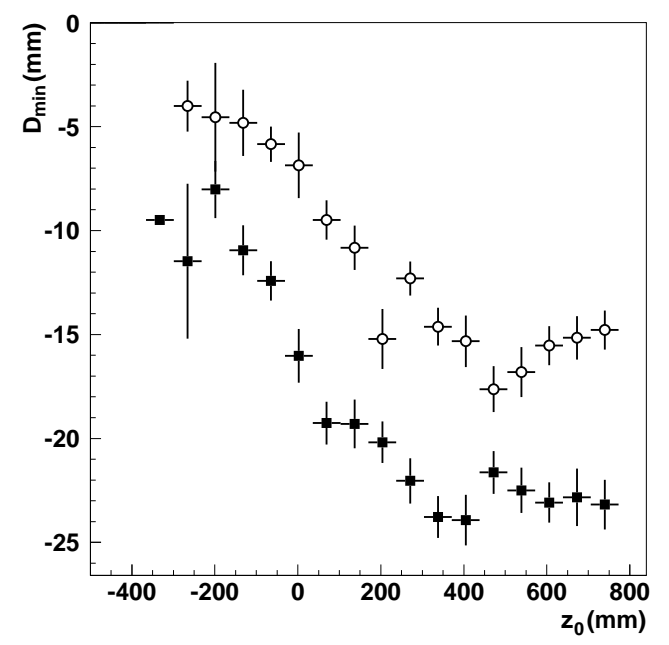

Fig. 8. The dependence of the distortion for cosmic-ray tracks at large $z$ on the position of ion cloud during the inter-spill period. Dmin is the minimum distance of the trajectory of one arm of the cosmic-ray track with respect to the point closest to the origin evaluated for the complete cosmic-ray track. Cosmic rays taken within the first $175 \mathrm{~ms}$ after the end of the spill are shown with closed circles, while the tracks taken between $250 \mathrm{~ms}$ and $300 \mathrm{~ms}$ after the end of the spill are shown with open circles. 

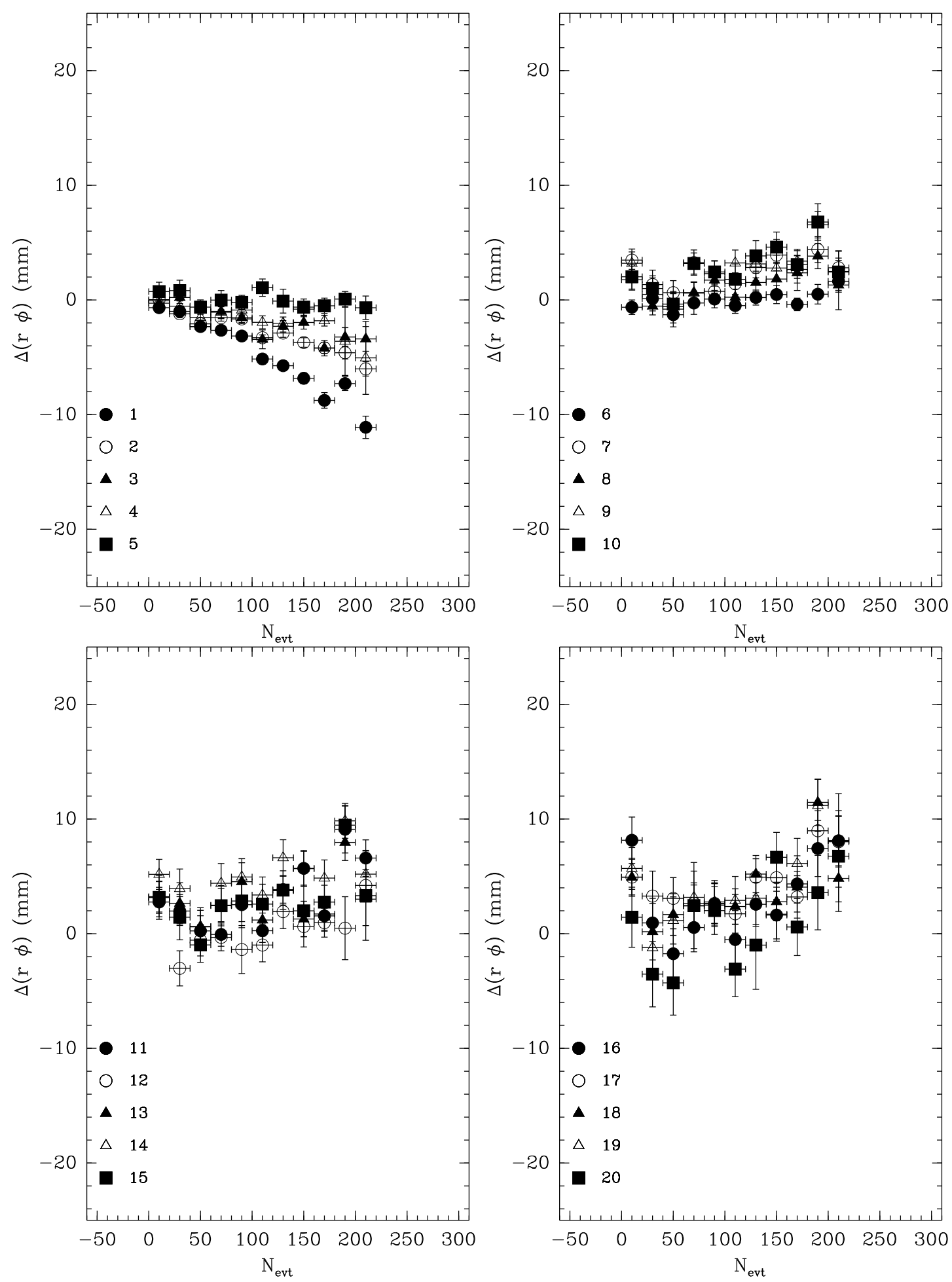

Fig. 9. The $R \phi$ distortion $\Delta(r \phi)=\left\langle(r \phi)_{\text {measured }}-(r \phi)_{\text {predicted }}\right\rangle$ measured row-by-row as a function of event number in spill for the $3 \mathrm{GeV} / c$ beam and $180 \mathrm{~cm}$ long $\mathrm{H}_{2}$ target data. The four panels show data for four groups of five pad-rows each. The different symbols represent the individual pad-rows. 

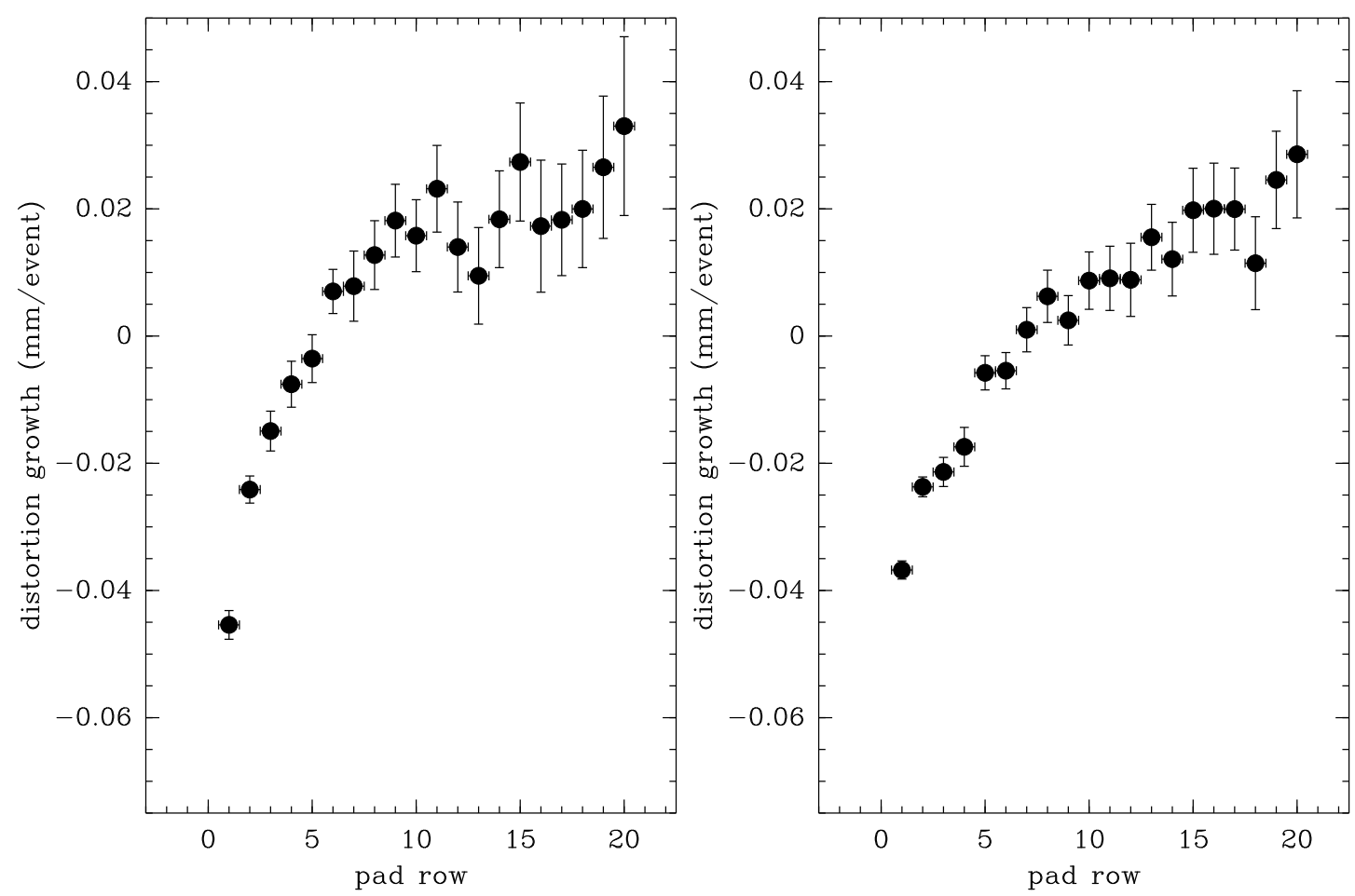

Fig. 10. The variation of the distortion strength as a function of the pad row number, fitted during the whole spill, left panel: $180 \mathrm{~mm} \mathrm{H}_{2}$ target, $3 \mathrm{GeV} / c$ beam; right panel: $60 \mathrm{~mm} \mathrm{H}_{2}$ target, $5 \mathrm{GeV} / c$ beam.

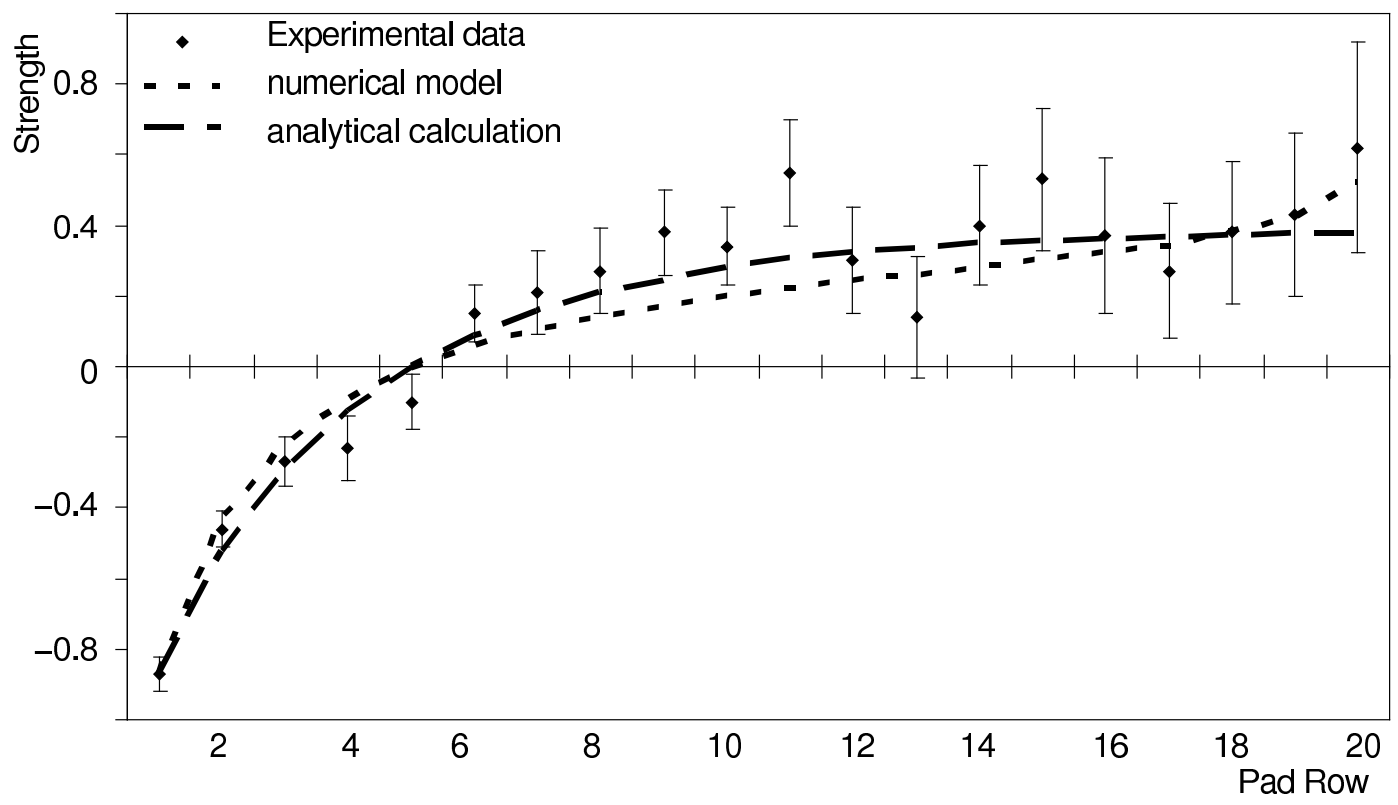

Fig. 11. The comparison of the measured distortion and the analytical models. The strength is shown with an arbitrary scale. Closed diamonds are the experimental data; long dashes show the analytical calculation for uniform space charge Eq. (7) and short dashes the numerical model. 

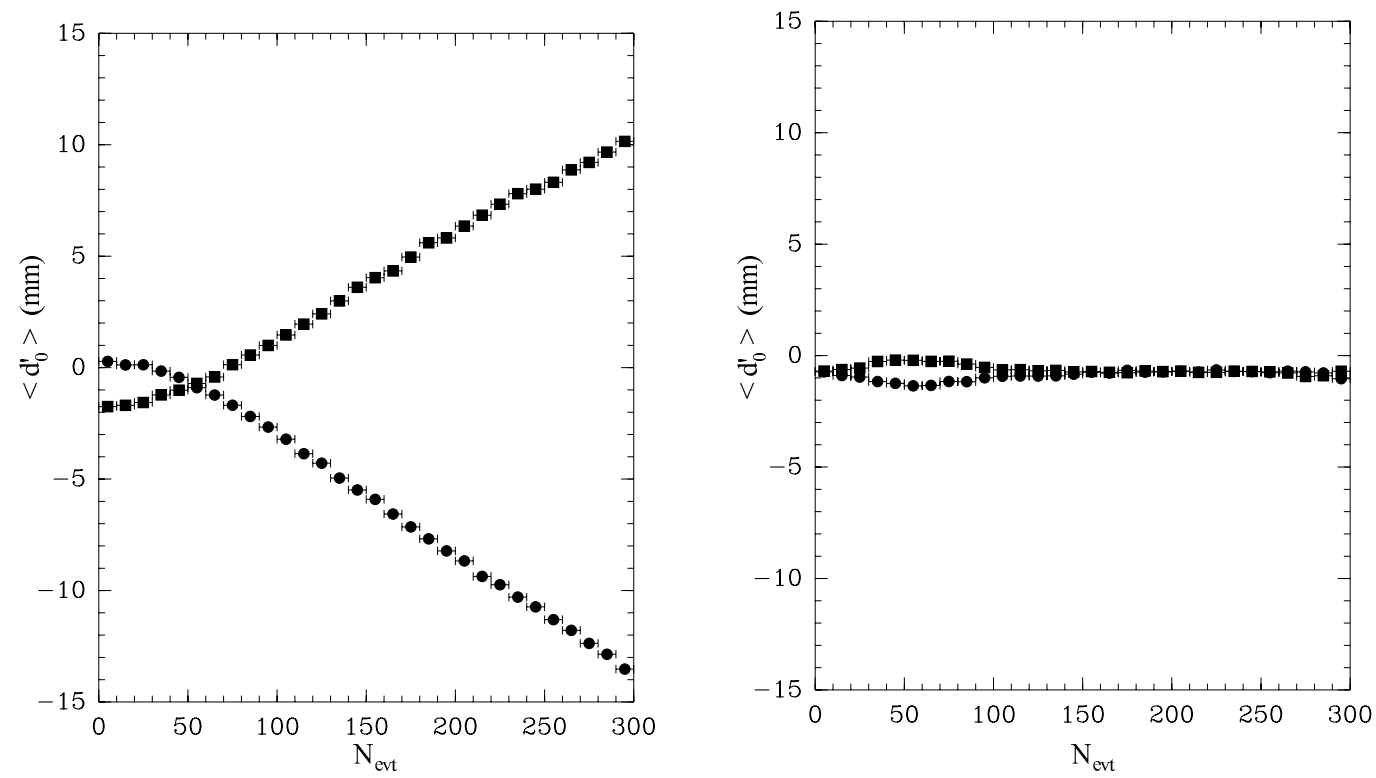

Fig. 12. Average $d_{0}^{\prime}$ (dots for reconstructed positive tracks, squares for reconstructed negative tracks) as a function of event number in spill for $8.9 \mathrm{GeV} / c$ Be data. (left panel uncorrected; right panel: dynamic distortion corrections applied.) After the "default" correction for the static distortions (equal for each setting) a small residual effect at the beginning of the spill is visible at $N_{\text {evt }}=0$ (left panel). This is due to the fact that the inner and outer field cages are powered with individual HV supplies. A setting-by-setting correction compatible with the reproducibility of the power supplies is applied for the data of the right panel together with the dynamic distortion correction. The value of $\left\langle d_{0}^{\prime}\right\rangle$ at $N_{\text {evt }}=0$ in the right panel has a small negative value as expected from the fact that the energy-loss is not described in the track-model used in the fit. The difference observed in the results for the two charges around $N_{\text {evt }}=50$ shows that even at the onset of the effect the model can correct the distortion within $1 \mathrm{~mm}$. 

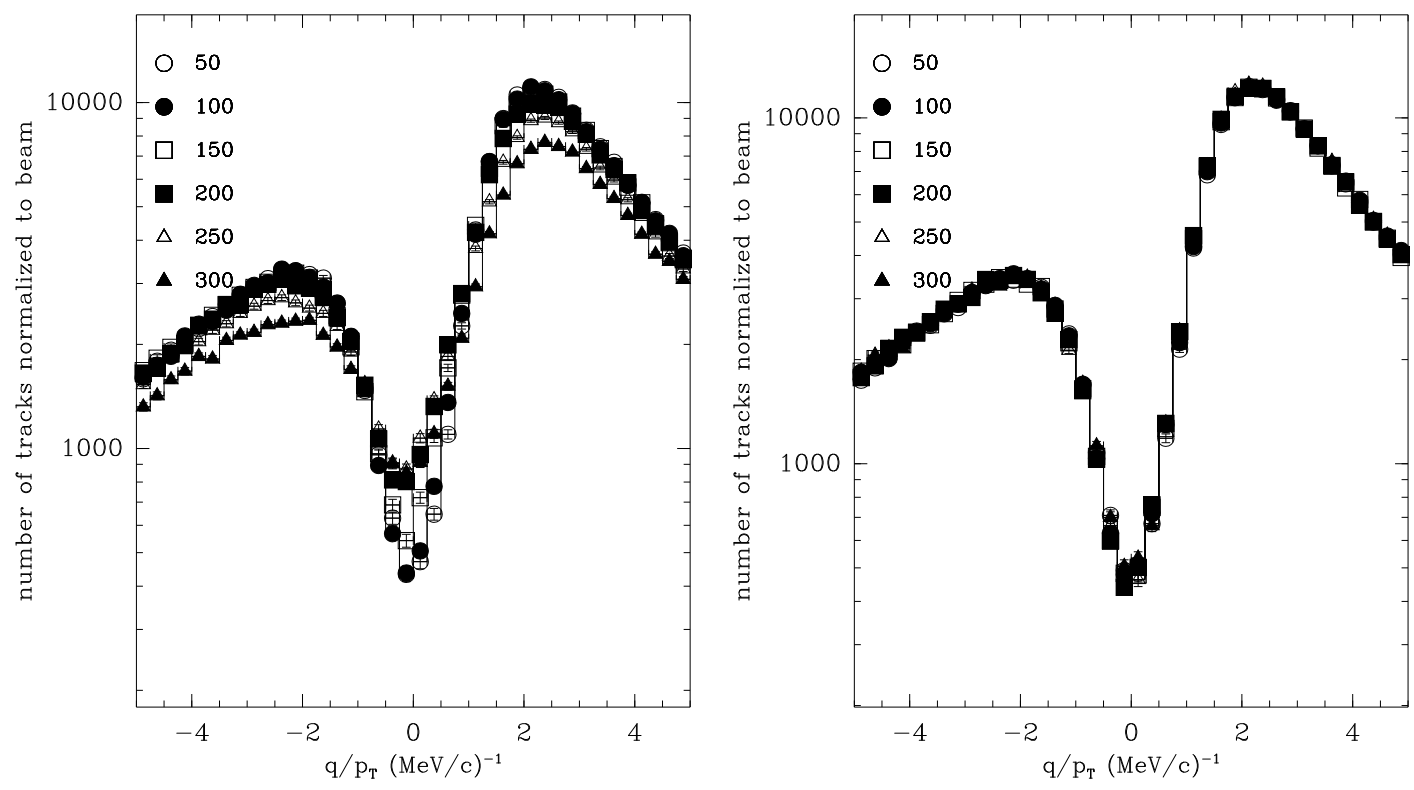

Fig. 13. Distribution in $1 / p_{\mathrm{T}}$ for the $8.9 \mathrm{GeV} / c$ Be data. The six curves show six regions in event number in spill (each in groups of 50 events in spill). Groups are labelled with the last event number accepted in the group, e.g. " 50 " stands for the group with event number from 1 to 50. The six groups are normalized to the same number of incoming beam particles, taking the first group as reference. Left panel: without dynamic distortion corrections; right panel: with dynamic distortion corrections. In the left panel only the first three groups of 50 events in spill are equivalent, while in the right panel all six groups are indistinguishable. 

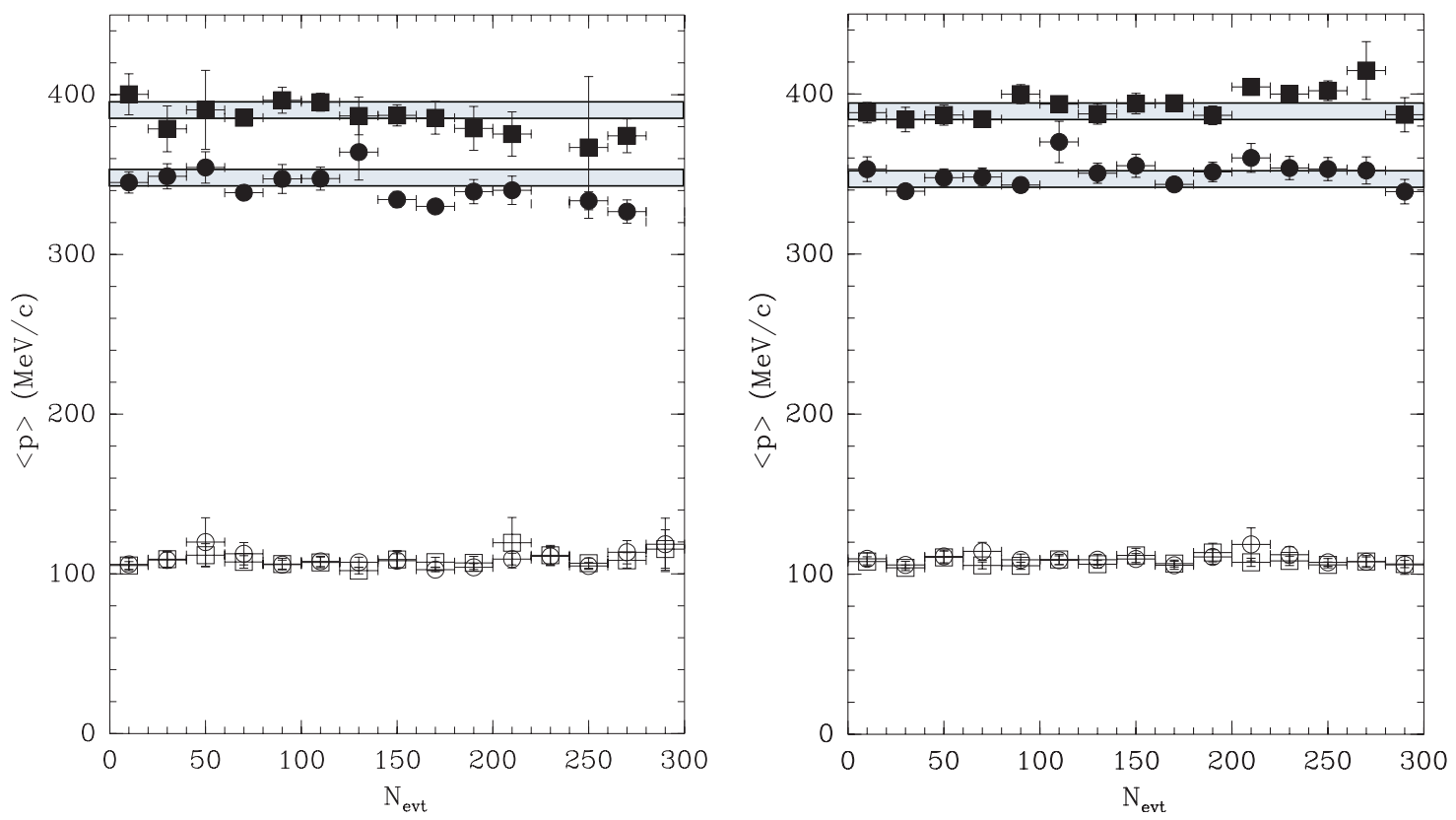

Fig. 14. Momentum benchmarks. Left panel uncorrected; right panel: dynamic distortion corrections applied. The closed boxes show the average momentum observed for protons selected using their range (reaching the second $\mathrm{RPC}$ ) and $\mathrm{d} E / \mathrm{d} x$; closed circles show protons selected within a high $\mathrm{d} E / \mathrm{d} x$ region; open circles: $\pi^{-}$selected with $\mathrm{d} E / \mathrm{d} x$; open boxes: $\pi^{+}$selected with $\mathrm{d} E / \mathrm{d} x$. The angle of the particles is restricted in a range with $\sin \theta \approx 0.9$. In the left panel (uncorrected data) one observes a variation of $\approx 5 \%$ for the high $p_{\mathrm{T}}$ samples. The corrected data stay stable well within $3 \%$. The shaded bands show a $\pm 1.5 \%$ variation. The low $p_{\mathrm{T}}$ data remain stable with or without correction. 


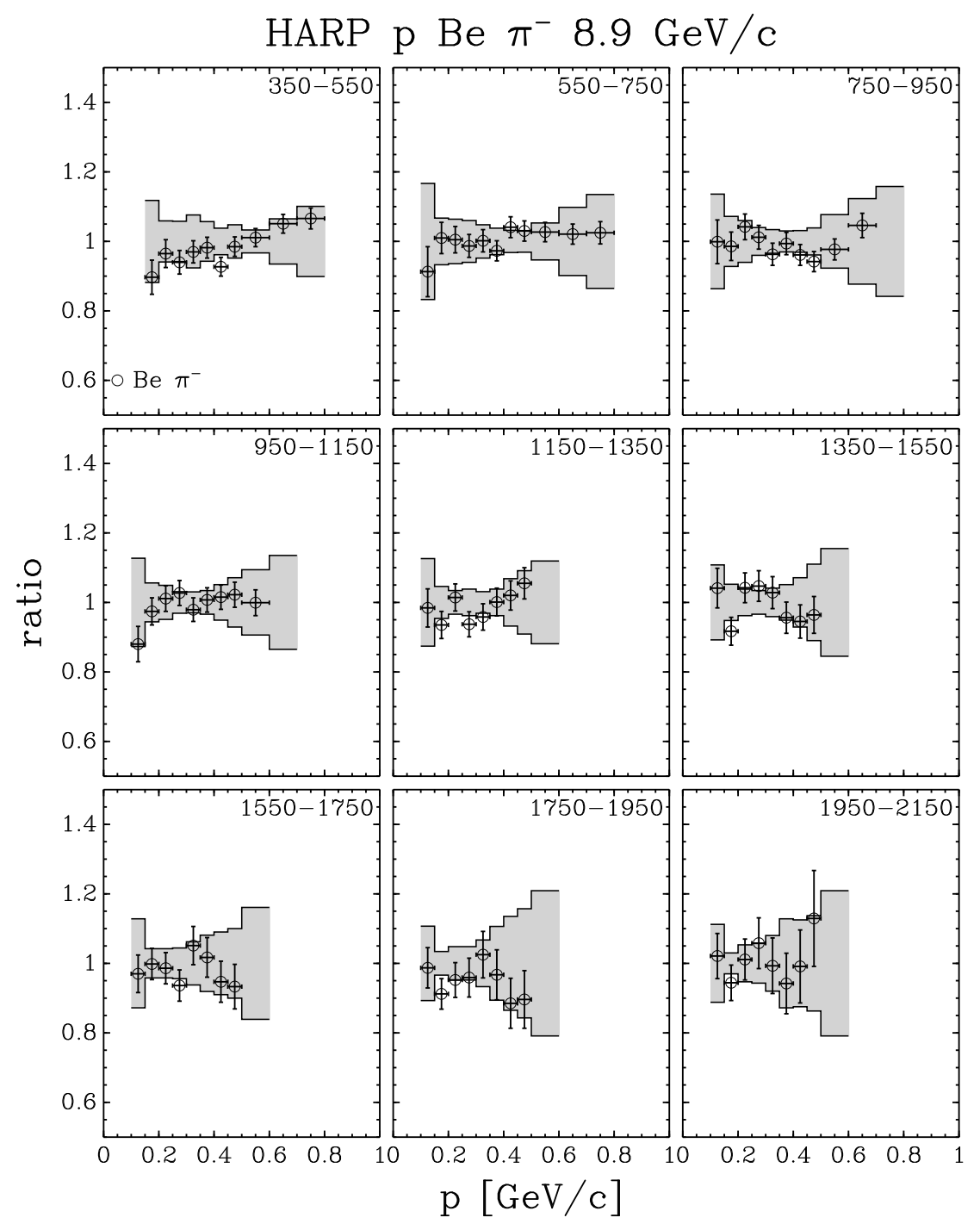

Fig. 15. Ratio of the $\pi^{-}$production cross-sections measured without and with corrections for dynamic distortions in $\mathrm{p}-$ Be interactions at $8.9 \mathrm{GeV} / c$, as a function of momentum shown in different angular bins (shown in mrad in the panels). The error band in the ratio takes into account momentum error and the error on the efficiency, the other errors being correlated. The errors on the data points are statistical. 


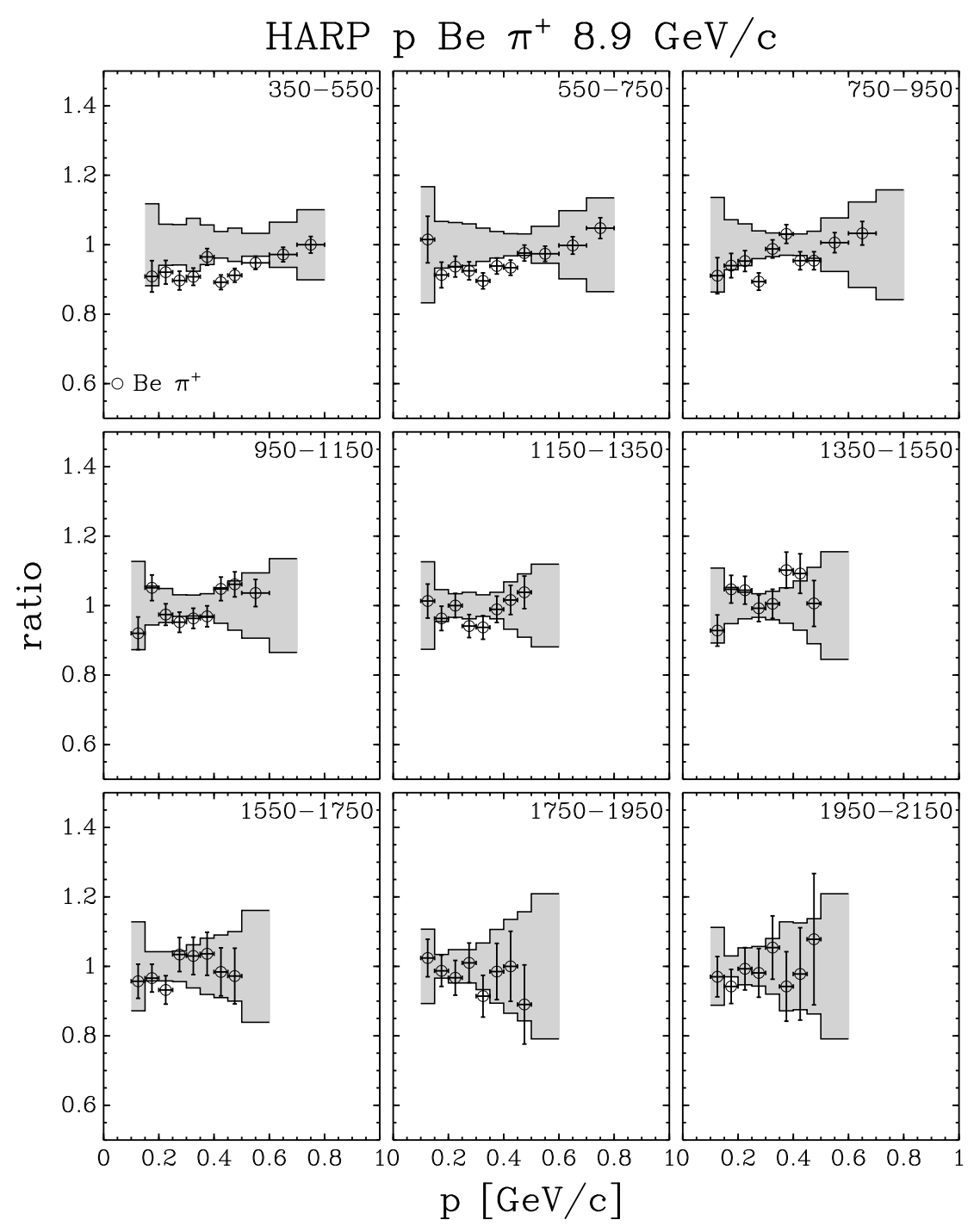

Fig. 16. Ratio of the $\pi^{+}$production cross-sections measured without and with corrections for dynamic distortions in $\mathrm{p}-$ Be interactions at $8.9 \mathrm{GeV} / c$, as a function of momentum shown in different angular bins (shown in mrad in the panels). The error band in the ratio takes into account momentum error and the error on the efficiency, the other errors being correlated. The errors on the data points are statistical. 